\title{
Review of: "LIO-CSI: LiDAR inertial odometry with loop closure combined with semantic information"
}

\author{
Ajay Kumar ${ }^{1}$ \\ 1 Daegu Gyeongbuk Institute of Science and Technology
}

Potential competing interests: The author(s) declared that no potential competing interests exist.

In this article Authors introduced semantic feature aware LiDAR Inertial Odometry (LIO) approach to reduce the map-based localization error by eliminating the dynamic objects from the point cloud registration process. Further, semantic feature information used to improve the LiDAR odometry and loop closure detection. This could be very interesting study, however there are few similar works already been introduced recently (Ex; [1] Dynamic Object Aware LiDAR SLAM based on Automatic Generation of Training Data, [2] LiDAR Odometry and Mapping Based on Semantic Information for Outdoor Environment). Any how existing LiDAR odometry approaches already proves that, integrating semantic information will enhance the performance compared to pure geometric method, and therefore this study have not much novality. There are no any scientific advancement found to claim novality. Apart from this, article satisfies all the requirements to publish at your journal.

Additionally, i would like to suggest the authors to include time analysis table to reflect the real time performance ability of proposed approach. Also, it would be more appropriate to provide comparison between the already existing semantic feature based lidar odometry approach along with current statistical analysis included in the article. 